\title{
Direct-write laser micromachining and universal surface modification of PMMA for device development
}

\author{
Ji-Yen Cheng $^{a, *}$, Cheng-Wey Wei ${ }^{\text {a,b }}{ }^{\text {, Kai-Hsiung Hsu }}{ }^{\text {a }}$, Tai-Horng Young ${ }^{b}$ \\ ${ }^{a}$ Institute for Applied Science and Engineering Research, 128 Sec. 2 Academia Road., Taipei 11529, Taiwan \\ ${ }^{\mathrm{b}}$ Institute of Biomedical Engineering, National Taiwan University, Taipei, Taiwan \\ Received 31 December 2002; received in revised form 21 October 2003; accepted 22 October 2003
}

\begin{abstract}
The use of direct-write laser micromachining on poly(methyl methacrylate) (PMMA) to fabricate microfluidic chip has the potential for fast prototyping and production. However, the advantage has been diminished by the rugged surface and the limited surface chemistry modification available. To overcome this problem, we have developed a flexible and economic pipeline including PMMA micromachining, surface smoothness improvement, and a universal surface modification for introduction of various functional groups. The micromachining is accomplished by a commercial laser scriber, to which a user designed computer drawing is sent directly for grooving. The typical trench width is $140 \mu \mathrm{m}$ while the aspect ratio up to more than 7 is easily achieved. Smooth surface is obtained after a one-step thermal annealing treatment after machining. The chemical modification is performed by a simple reduction reaction followed by the widely used organosilane chemistry to introduce diverse functional groups such as perfluoroalkyl $\left(-\mathrm{C}_{n} \mathrm{~F}_{2 n+2}\right)$, amino $\left(-\mathrm{NH}_{2}\right)$, and sulfhydryl (-SH) for surface passivation or further biomolecule immobilization. The surface smoothness after thermal annealing has been examined by scanning electron microscopy (SEM) and atomic force microscopy (AFM) to be comparable to pristine surface. The surface chemistry modification has been confirmed by attenuated total reflectance-Fourier transform infrared spectroscopy (ATR-FTIR) and X-ray photoelectron spectroscopy (XPS). The whole procedure provides a very efficient micromachining platform to fabricate PMMA microfluidic chip for both prototyping and production.
\end{abstract}

(C) 2003 Elsevier B.V. All rights reserved.

Keywords: Laser-micromachining; PMMA modification; Microfluidic biochip

\section{Introduction}

A technology platform for both flexible prototyping and efficient mass production is usually hard to achieve. For example, the research of micrototal analysis system (microTAS, or lab-on-a-chip technology) that receives intensive attention recently is often confronted with such dilemma. When matured semiconductor processing technology is borrowed to fabricate a microfluidic analysis system, the time-consuming photomask generation, photolithography, and etching process hampers the rapid turnaround of new design although the technology intrinsically suits for mass production.

To reduce the prototype development effort, we use a direct-write laser machining system (Scheme 1) to fabricate microfluidic channels on poly(methyl methacrylate)

\footnotetext{
* Corresponding author. Tel.: +886-2-2789-8000; fax: +886-2-2782-6680.

E-mail address: jycheng@gate.sinica.edu.tw (J.-Y. Cheng).
}

(PMMA) substrate. The user designs patterns using common drawing tools such as CorelDraw or AutoCAD. The pattern is then sent to the system for automatic machining. In this system, the $\mathrm{CO}_{2}$ laser beam is directed with two-dimensional robotic arms to move across the entire machining area of $31 \mathrm{~cm} \times 61 \mathrm{~cm}$. The trench is machined by the ablation process when the laser is focused on the plastic substrate. Variable trench depth can be fabricated by adjusting the laser power, beam motion speed, or number of passes of laser. The third-dimensional robotic motion allows the user to change the beam spot size to fabricate trenches with different widths.

The use of PMMA as the substrate material has several advantages. The PMMA sheet is a non-porous solid and therefore contamination caused by biomolecule adsorption is diminished. In addition, PMMA is inert in neutral aqueous solution and no hydrolysis occurs during the application. The manufacturing process of PMMA microfluidic chips has received much attention. Several fabrication methods such as hot embossing [1], wire imprinting [2], and excimer 


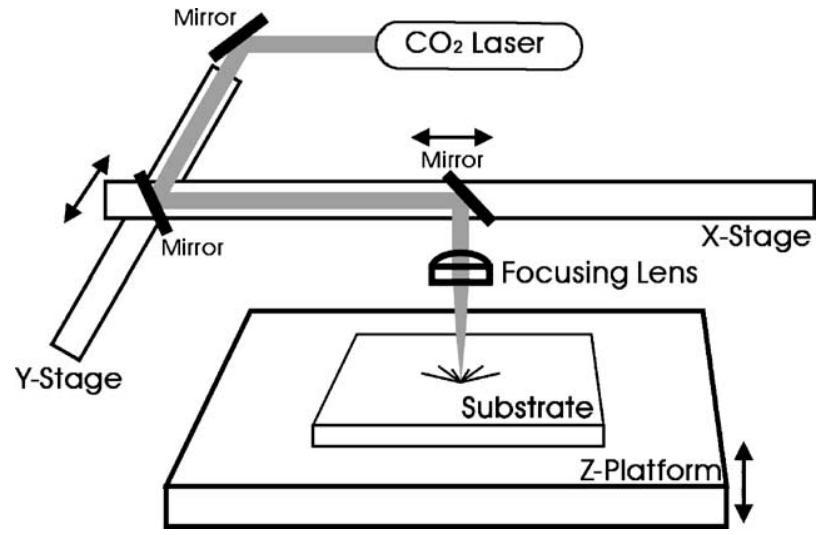

Scheme 1. Configuration of a direct-write laser machining system with an $X-Y$ stage.

laser ablation [3] have been studied. However, very rare results describing PMMA micromachining using $\mathrm{CO}_{2}$ laser has been reported. Very recently, a laser machining method using swivel mirror for $100 \mathrm{~mm} \times 100 \mathrm{~mm}$ PMMA substrate has been reported [4]. However, the problem of rough surface after laser machining persists.

The carbon skeleton of the polymer, PMMA, consists of a saturated $\mathrm{C}-\mathrm{C}$ backbone with dangling ester groups, extending $1-2 \AA$ from the backbone. The typical reaction of the ester group, like other carboxylic acid derivatives, is nucleophilic substitution. For example, the hydrolysis and aminolysis convert the ester group into its constitutional organic acid and amide derivatives, respectively [5]. The aminolysis of surface bound ester group has been applied for PMMA surface modification [6]. Since the surface bound functional group is usually less reactive than that in its solution phase, lithioamine rather than primary amine is used for aminolysis to accomplish the task. The former has higher basicity and hence higher nucleophilicity to drive the PMMA surface chemistry. In order to maintain its basicity, extensive solvent drying procedures are required during the reaction.

In this work, we have developed a flexible and economic pipeline for PMMA micromachining, surface smoothness enhancement and a universal chemical modification. In addition to physical modification to smoothen the rugged surface after laser machining, we also describe a PMMA surface modification for convenient incorporation of versatile functional groups. After initial introduction of surface hydroxyl group, organosilanes can be readily applied for further introduction of various groups such as amino group $\left(-\mathrm{NH}_{2}\right)$, perfluoroalkyl group $\left(-\mathrm{C}_{n} \mathrm{~F}_{2 n+2}\right)$, and sulfhydryl group (-SH) (Scheme 2). The modified surface can be used for the immobilization of biomolecules such as in the applications of DNA $[7,8]$ and protein microarray $[9,10]$. The introduction of other functional groups can also be performed similarly by the use of corresponding organosilanes.

PMMA

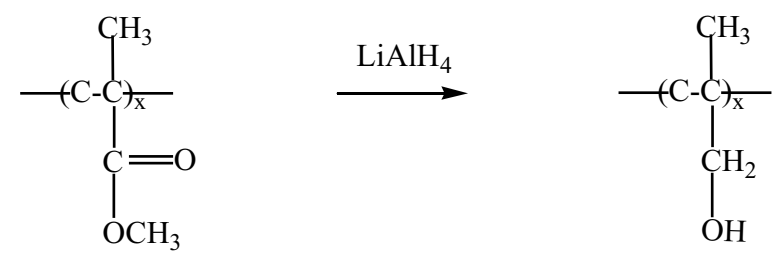

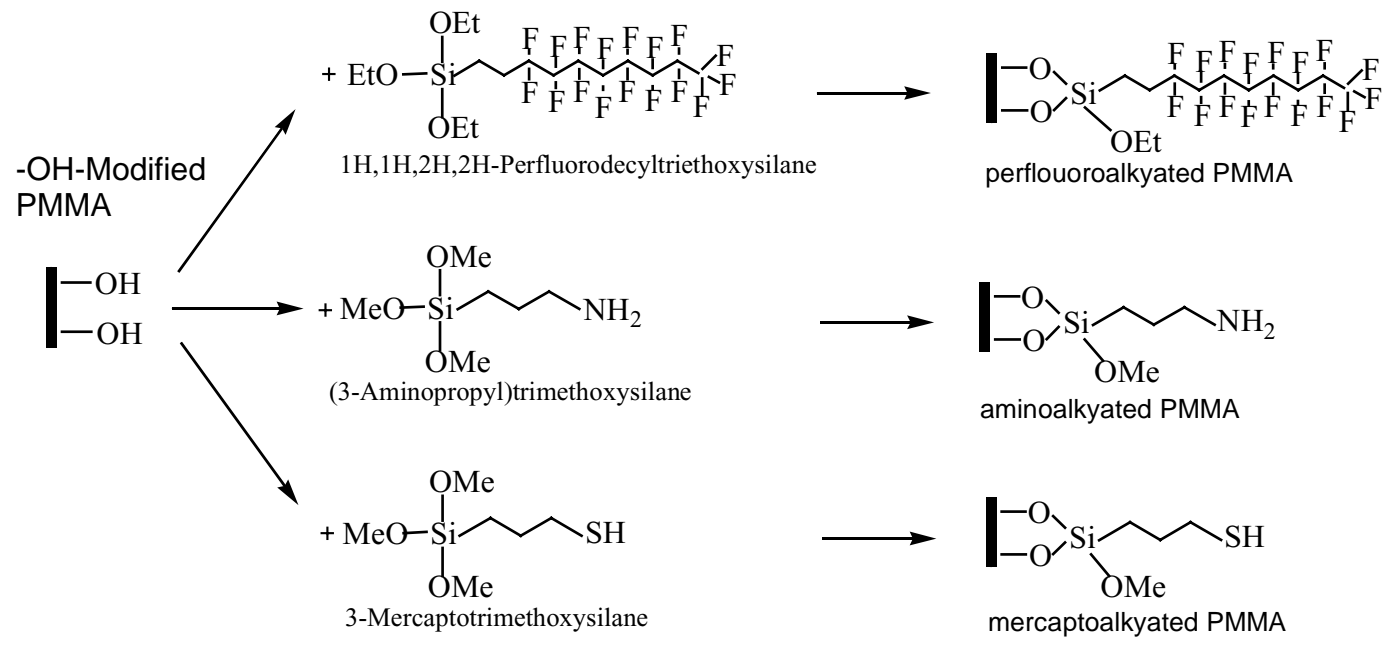

Scheme 2. A universal modification strategy for PMMA. Chemical reduction of PMMA surface by LAH (a) and further functional group introduction by various organosilanes: perfluoroalkylation, aminoalkylation, and mercaptoalkylation (b). 


\section{Experimental}

\subsection{Chip fabrication}

A commercial laser scriber (M-300, Universal Laser Systems) is used to engrave the PMMA substrate. The $\mathrm{CO}_{2}$ laser has wavelength of $10.6 \mu \mathrm{m}$ and its full power is $25 \mathrm{~W}$. The configuration in Scheme 1 shows a focused laser beam that can scan over a two-dimensional area by the combined motion of $X$ and $Y$ stages. The maximal speed of the beam motion is $600 \mathrm{~mm} / \mathrm{s}$. The substrate is secured on a platform that can move in the $Z$-axes. The microfluidic pattern is designed using CorelDraw (Corel) or AutoCAD (Autodesk). The pattern is then sent to the laser scriber for direct machining on PMMA substrate. To form a channel, the trench is sealed by thermally bonding the patterned substrate with a blank PMMA substrate at $120^{\circ} \mathrm{C}$ for $30 \mathrm{~min}$. Additional thermal annealing process at $170^{\circ} \mathrm{C}$ for $30 \mathrm{~min}$ is performed to enhance the surface smoothness.

\subsection{Surface modifications}

The surface modification is performed as follows. The cast PMMA sheet is obtained from a local supplier (Chung Jih Plastic Co. Ltd., Taiwan) and the extruded PMMA is obtained from RS Components Ltd. (cat. no. 824-480). The sheet is cut and then sonicated in 2-propanol (Fluka), rinsed with deionized water (resistivity $18.2 \mathrm{M} \Omega \mathrm{cm}$, Millipore) and dried in a stream of dry nitrogen. The cast PMMA is then put in lithium aluminum hydride (LAH, Acros) solution in diethyl ether (J.T. Baker) for $24 \mathrm{~h}$. An amount $16 \mathrm{~g}$ of LAH in $100 \mathrm{ml}$ ether is used for the chemical reduction. The solution is constantly agitated during reaction. For the incorporation of organosilanes, the cast PMMA sheet is first cleaned thoroughly by sonication in 2-propanol. The sheet is then immersed in the solution of $1 \mathrm{H}, 1 \mathrm{H}, 2 \mathrm{H}, 2 \mathrm{H}$-perfluorodecyltriethoxysilane (Lancaster), 3-(aminopropyl)trimethoxysilane (Fluka), or 3 -mercaptotrimethoxysilane (Aldrich) in 2-propanol $(5 \%$ $\mathrm{v} / \mathrm{v})$ for $1 \mathrm{~h}$. The sheet is then rinsed thoroughly with 2-propanol and then cured in a vacuum oven at $80^{\circ} \mathrm{C}$ for $16 \mathrm{~h}$.

\subsection{Surface analysis}

The contact angle is measured as follows. An amount of $10 \mu l$ of deionized water is applied onto the substrate surface. The contact angle is then determined by the image of the water drop. A Leo 1530 field emission scanning electron microscope (SEM, Leo, UK) is used to measure the cross section of the channel. Except for initially machined PMMA and extruded substrate, all SEM pictures are taken after thermal annealing. The atomic force microscopic (AFM) image for the measurement of surface roughness is collected on a Solver P-47 (NT-MDT, Zelenograd, Russia) scanning atomic microscope. The microscope is operated in force modulation mode for all experiments. The attenuated total reflectance-Fourier transform infrared (ATR-FTIR) spectra are obtained using an IR-Plan Advantage Microscope (Spectra Tech, USA) attached to an Excalibur FTS-3000 spectrometer (Bio-Rad, USA). The substrate is subjected to hot baking at $80^{\circ} \mathrm{C}$ for $24 \mathrm{~h}$ to remove surface bound water. Each spectrum represents the average of 256 scans in $4 \mathrm{~cm}^{-1}$ resolution. The X-ray photoelectron spectra are collected on an ESCALAB 250 (VG Scientific, UK) spectrometer using XR5 monochromatic X-ray gun with a power of $300 \mathrm{~W}$. The spectrum is an average of three scans with $2 \mathrm{eV}$ per step and with pass energy of $30 \mathrm{eV}$. The dwell time is $100 \mathrm{~ms}$. The binding energy of carbon and oxygen in PMMA are 286.0 and $534.0 \mathrm{eV}$, respectively.

\section{Results}

A sample pattern to demonstrate the machining flexibility is shown in Fig. 1. The sample shows various channel widths ranging from 140 to $900 \mu \mathrm{m}$. The straight channel, $\mathrm{U}$ turns, merging and branching channels, wide channel, and spiral are all designed in the same computer drawing. The channel is filled with dye for visual inspection. The minimal width is $100 \mu \mathrm{m}$. The width of the wide channel is $860 \mu \mathrm{m}$. The depth of the channel can be adjusted by the laser power, the motion speed, or number of pass of laser beam used in scribing. These parameters are designed by the user's drawing and are controlled by the computer when in machining. The width of the channel is controlled by varying the beam size. When the substrate is positioned at beam focusing plane, the minimal width is obtained. By moving the substrate away from the focal plane, a large channel width is achieved. The cross sections of the trenches with various widths and depths are shown in Fig. 2. The beam scanning speed is $36 \mathrm{~mm} / \mathrm{s}$. Trenches with high aspect ratios can be easily machined. Fig. 3 shows the picture of a sealed channel in cast PMMA. No distortion of the channel profile is observed. Three pictures comparing the distorted rims of the carved trenches are shown in Fig. 4 for cast and extruded PMMA sheet. Clear bulges on the rim are observed for extruded PMMA.

The surface of the laser-machined trench wall is initially very rugged as shown in Fig. 5(a). The SEM picture of the smooth surface after thermal annealing is shown in Fig. 5(b). The inset shows the AFM topography. The surface of the initially machined trench wall is so rugged that AFM measurement cannot be performed.

The surface quality of LAH treated PMMA substrate is transparent and smooth by visual inspection. The smoothness for the pristine and chemically reduced PMMA substrate is measured by AFM and shown in Fig. 6(a) and (b), respectively. No obvious change in surface quality is observed. 


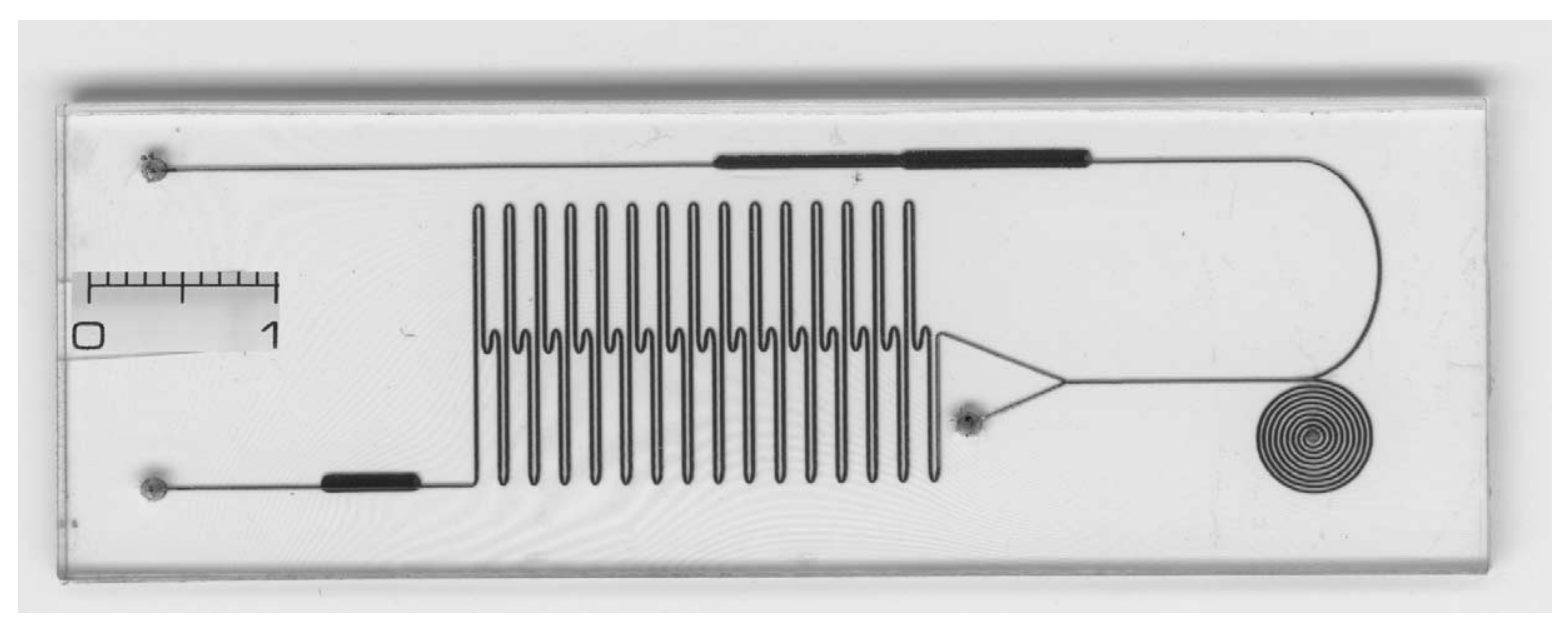

Fig. 1. A sample pattern showing the various pattern fabricated simultaneously in the same PMMA substrate. The scale bar is $1 \mathrm{~cm}$.

The effect of chemical reduction is examined by contact angle change, ATR-FTIR, and XPS. The ATR-FTIR spectra of pristine and LAH treated PMMA surface are shown in Fig. 7 (a) and (b). The contact angle change from $68.4^{\circ}$ for the pristine surface to $32.7^{\circ}$ for the modified surface is shown in the photo. The peak characteristic of hydroxyl group $(-\mathrm{OH})$ in Fig. 7(b) is consistent with the chemical reduction, which produces alcohol as one of the products.
The ability to carry out the silanization reaction on the LAH treated surface was investigated by XPS. Fig. 8(a)-(d) show the XPS spectra of several organosilane modified PMMA substrates. The pristine substrate does not allow the silanization reaction and hence no silicon $(\mathrm{Si})$ peak can be observed in Fig. 8(a). In contrast, clear fluorine (F), nitrogen $(\mathrm{N})$, and sulfur (S) peaks can be observed in Fig. 8(b)-(d), respectively.

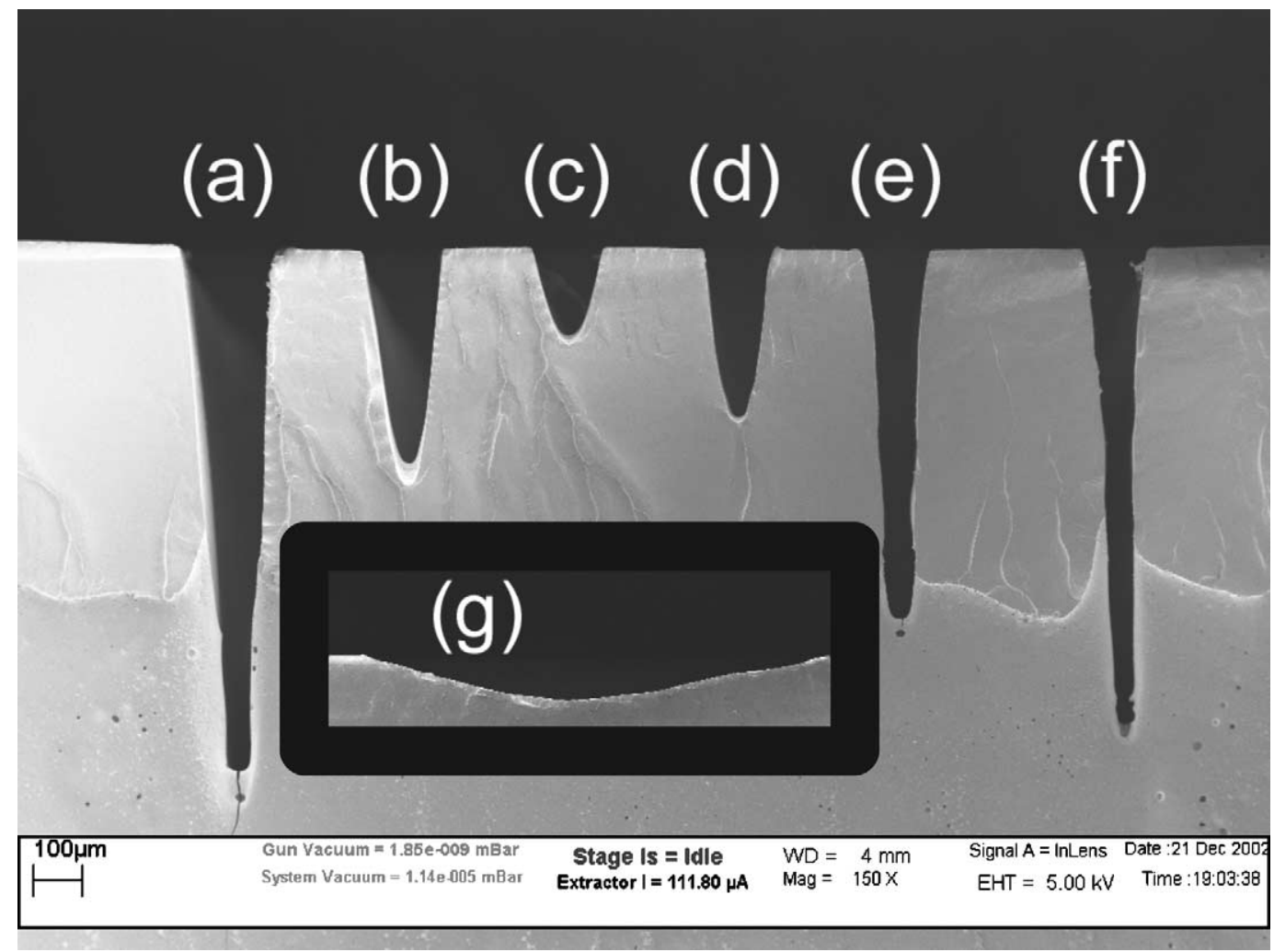

Fig. 2. Trenches in cast PMMA carved with various laser power and passes. The (a), (b), and (c) grooves are machined with laser power of 7.5, 3, and $1.5 \mathrm{~W}$, respectively. The (d), (e), and (f) are carved with $1.5 \mathrm{~W}$ laser power but with 2 , 5 , and 10 passes, respectively. The inset shows a wide-shallow groove carved with $1.5 \mathrm{~W}$ laser power and at speed of $36 \mathrm{~mm} / \mathrm{s}(\mathrm{g})$. The samples are subjected to thermal annealing before taking the pictures. 


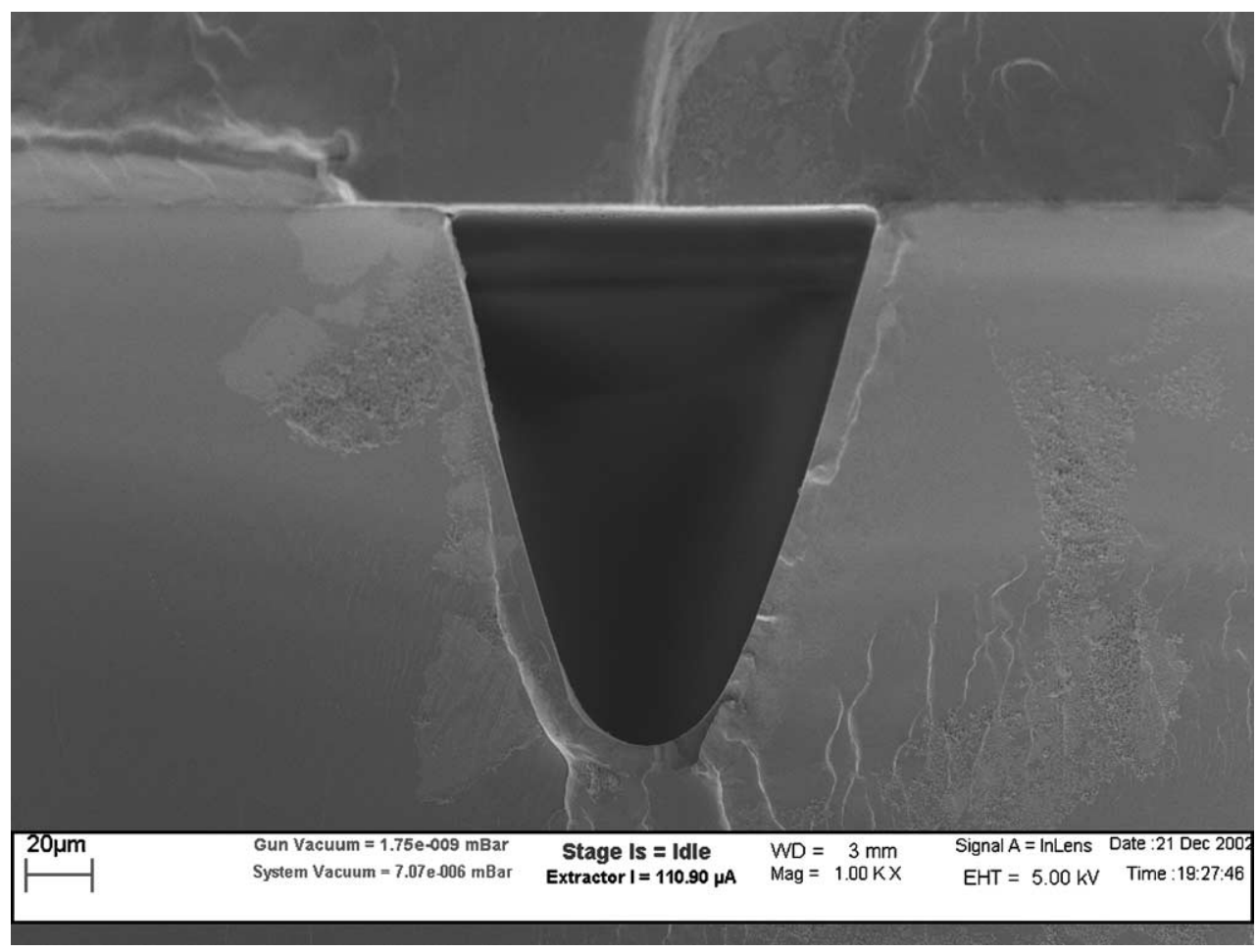

Fig. 3. The close-up of a sealed channel carved with $1.5 \mathrm{~W}$ laser power and at speed of $36 \mathrm{~mm} / \mathrm{s}$. The samples are subject to thermal annealing before taking the pictures.

\section{Discussion}

\subsection{Flexible pattern fabrication and possible applications}

There are several advantages using the direct-write laser-machining method with the configuration shown in Scheme 1. First of all, the overall development time for a microfluidic chip is largely reduced since a desired pattern can be generated and put to work in hours instead of weeks. Second, channels with varying aspect ratios can be easily fabricated in the same substrate. Complex microfluidic patterns can be fabricated without difficulties. Third, the large machining area allows production in large quantity. For example, using the machining area of $31 \mathrm{~cm} \times 61 \mathrm{~cm}, 450$ copies of a $2 \mathrm{~cm} \times 2 \mathrm{~cm}$ pattern can be manufactured at the same time in an hour. Thousands of copies can be produced in one workday using one system. Since the system is inexpensive, further extension in production flexibility and capacity can be easily achieved by employing more systems. Therefore, the throughput of this system is adequate for both prototyping and production. Another advantage of the $X-Y$ stage is that the laser beam is perpendicular to the substrate at all times when using the $X-Y$ stages to move the laser beam across the substrate. Furthermore, the distance from the focusing lens to the substrate is kept constant when laser beam moves. Therefore, the beam spot size on the substrate remains unchanged and the trench cross section is consistent across the entire machining area. The benefit is not shared with a design using a swivel mirror to steer the laser beam across the substrate for machining, especially when the machining area is large.

The possible application for each component in the sample pattern (Fig. 1) is discussed briefly as follows. The straight channel can be used to transport sample between components. The area with U-turns is intended for applications such as heat transfer, e.g. polymerase chain reaction (PCR) in continuous format [11]. The merging and branching region connected to the right side of the U-turns can be utilized for diffusion reactions, e.g. extraction or enzymatic reaction [12]. The spiral can be used to extend the separation length in a limited area [13]. The wide channel can be used for a buffer reservoir or for retention of separation media such as reversed-phase resin. The resin can be blocked to avoid dislocation using a section of shallow channel functioning as a weir. The thus packed resin can be used for chromatographic separation such as sample preparation for matrix assisted laser desorption ionization (MALDI) and electrospray ionization (ESI). Several through-holes for reagent inlets and outlets are also machined onto the microfluidic chip at the channel termini. The opening diameter is defined in the computer drawing.

In spite of the complexity of the pattern shown in Fig. 1, it can be re-designed, machined and tested in less than an hour. Using a common drawing software, the pattern can be modified easily. The sample pattern can be generated in $2 \mathrm{~min}$ using the machining system. For preliminary tests and applications without high pressure, adhesive tape can be used to seal the trench to save the process time. Otherwise, thermal 

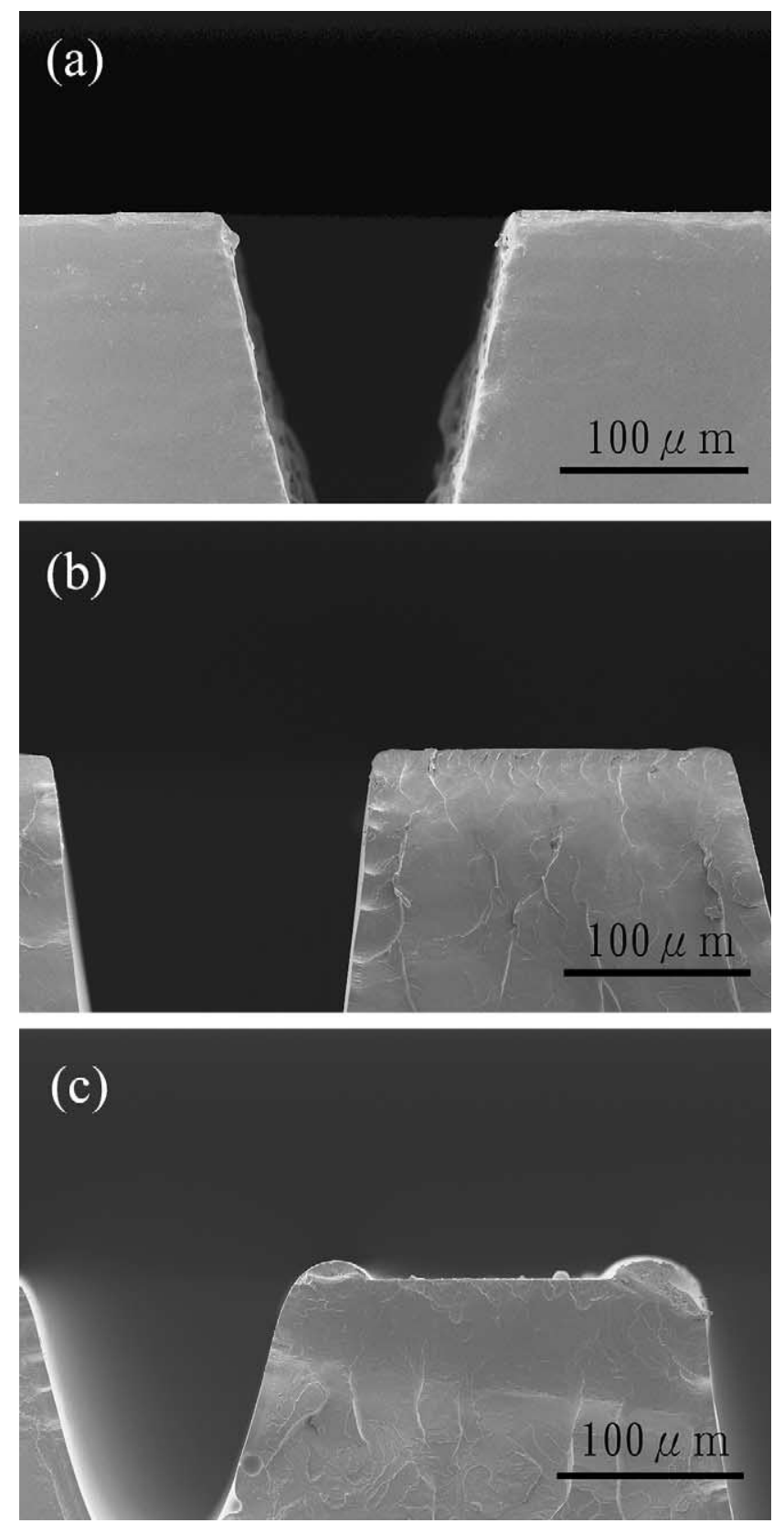

Fig. 4. The comparison of the groove rim with cast PMMA without annealing (a) cast PMMA with annealing (b), and extruded PMMA without annealing (c).

bonding provides secure sealing and can resist pressures up to 300 psi.

\subsection{Surface profile of laser-machined grooves-cross section and smoothness}

The cross section of the laser-machined groove resembles a Gaussian function. Since the intensity profile of the laser beam is Gaussian because it is operated in $\mathrm{TEM}_{0,0}$ mode, this resemblance is reasonable. The depth of the machined groove can be controlled by either laser power, mo- tion speed, or number of passes over the substrate. Although higher power and number of passes show a similar effect in deepening the grooves, the effects are not the same. As can be seen from Fig. 2, multiple passes of the laser beam do not increase the groove depth in a linear relationship. On the contrary, power multiplicity deepens the groove linearly but the width also increases with increasing power although the cross section still resembles a Gaussian. The width of the grooves increase from $144 \mu \mathrm{m}$ at $1.5 \mathrm{~W}$ laser power to 168 and $193 \mu \mathrm{m}$ when the power is increased two and five folds, respectively ((c), (b), and (a), Fig. 2). However, the width stays constant when a deep trench is carved by multiple passes of laser. Although the depth increases with increasing passes, the cross section starts to deviate from Gaussian for trenches with more than five passes ((e) in Fig. 2). It is possible that the ablated methyl methacrylate (MMA) re-deposits on the side wall and causes the deviation. The reason why this phenomenon is not observed in trenches carved with high power (Fig. 2(a)) may be that the ablated MMA vapor absorbs the laser power before it can re-deposit on trench wall and eventually escapes the trench. Similar dependence of the depth on the laser power has been observed by other research groups although our results show a typical width of $140 \mu \mathrm{m}$ [4]. Since the wavelength of the $\mathrm{CO}_{2}$ laser is $10.6 \mu \mathrm{m}$ the typical width we have obtained is larger than the diffraction limit. The minimal width may be reduced with a modification of the optical system.

Using this machining system, aspect ratios larger than 7 can be easily achieved. In Fig. 2, the aspect ratio is 1.2, 2.4, 5.2, and 7.0 with $1,2,5$, and 10 passes, respectively (trench (c), (d), (e), and (f)). An higher aspect ratio is possible as long as the PMMA can sustain the structure from collapsing. The high-aspect-ratio structure may be used to enlarge channel volume without increasing the channel width while the shallow channel can be used for particle retention. For example, channel depth smaller than the separation resin bead diameter can be used to secure the resin such as in the applications of solid phase extraction using microseparation column on chip $[14,15]$. However, further surface chemistry modification is needed for such applications.

The cross section remains unchanged after thermal annealing and bonding. The SEM picture in Fig. 3 shows that the thermal processes do not affect the cross section of the channel. The straight boundary between the two PMMA sheets indicates that the refill on the rim does not occur.

Distinguished by the preparation method, there are currently two types of PMMA sheet available commercially. They are cast and extruded PMMA. The cast PMMA has higher softening temperature than that of the extruded PMMA. We have tried the performance of both types of PMMA. Although the roughness is reduced when extruded PMMA sheet is used in machining, occasional bulges are still present (data not shown). The cast PMMA shows a rougher surface after machining. However, the cross section distortion at the rim is negligible. The comparison shown in Fig. 4 indicates that a clear bump occurs at the trench 

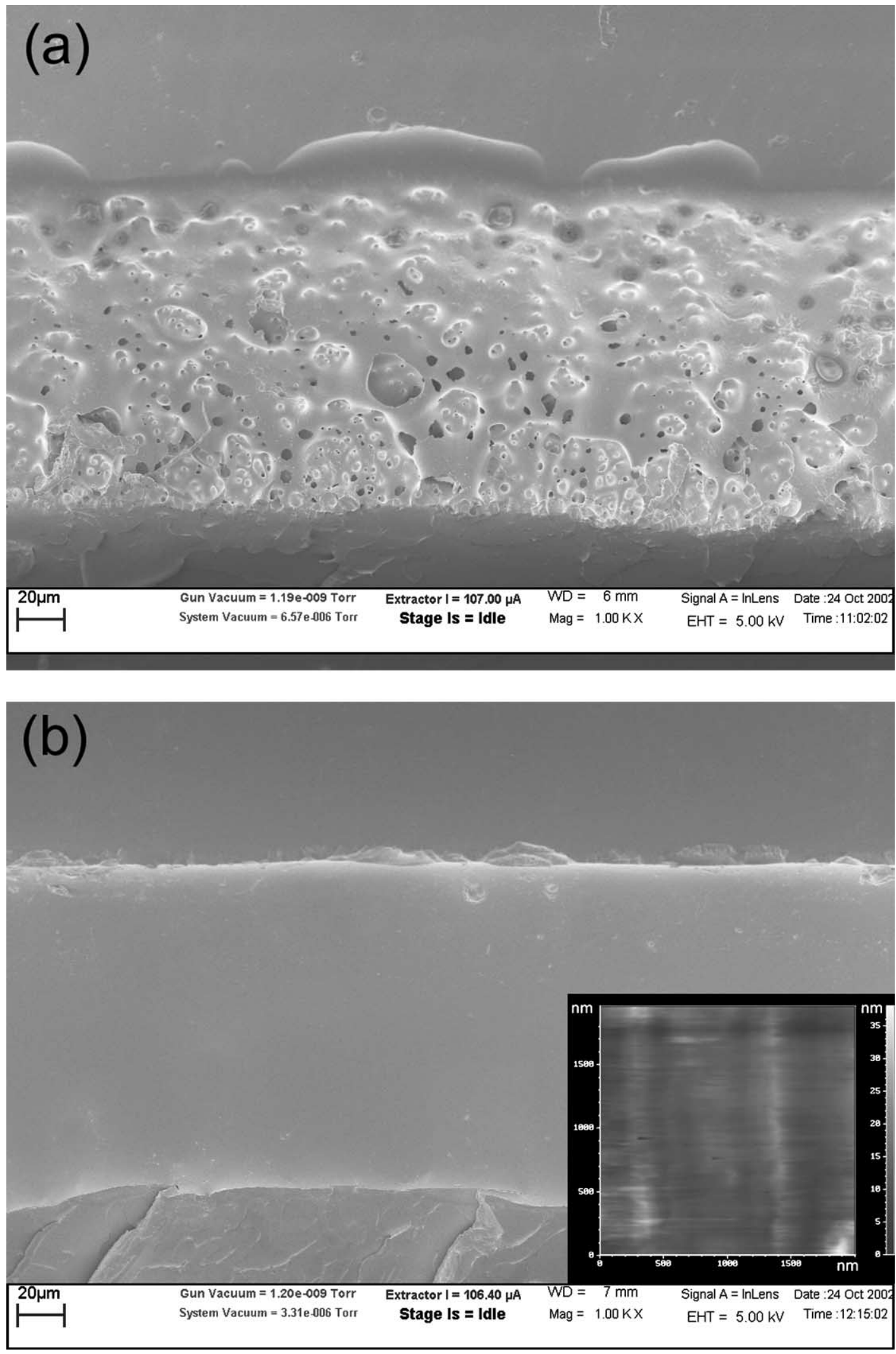

Fig. 5. The SEM pictures showing the rugged interior surface of the trench after laser machining (a) and smooth surface after thermal annealing (b). The AFM topography of the annealed surface is shown in the inset with full scale of $38 \mathrm{~nm}$ in the $Z$-axes. The viewing angle is perpendicular to the plane of the side wall. 


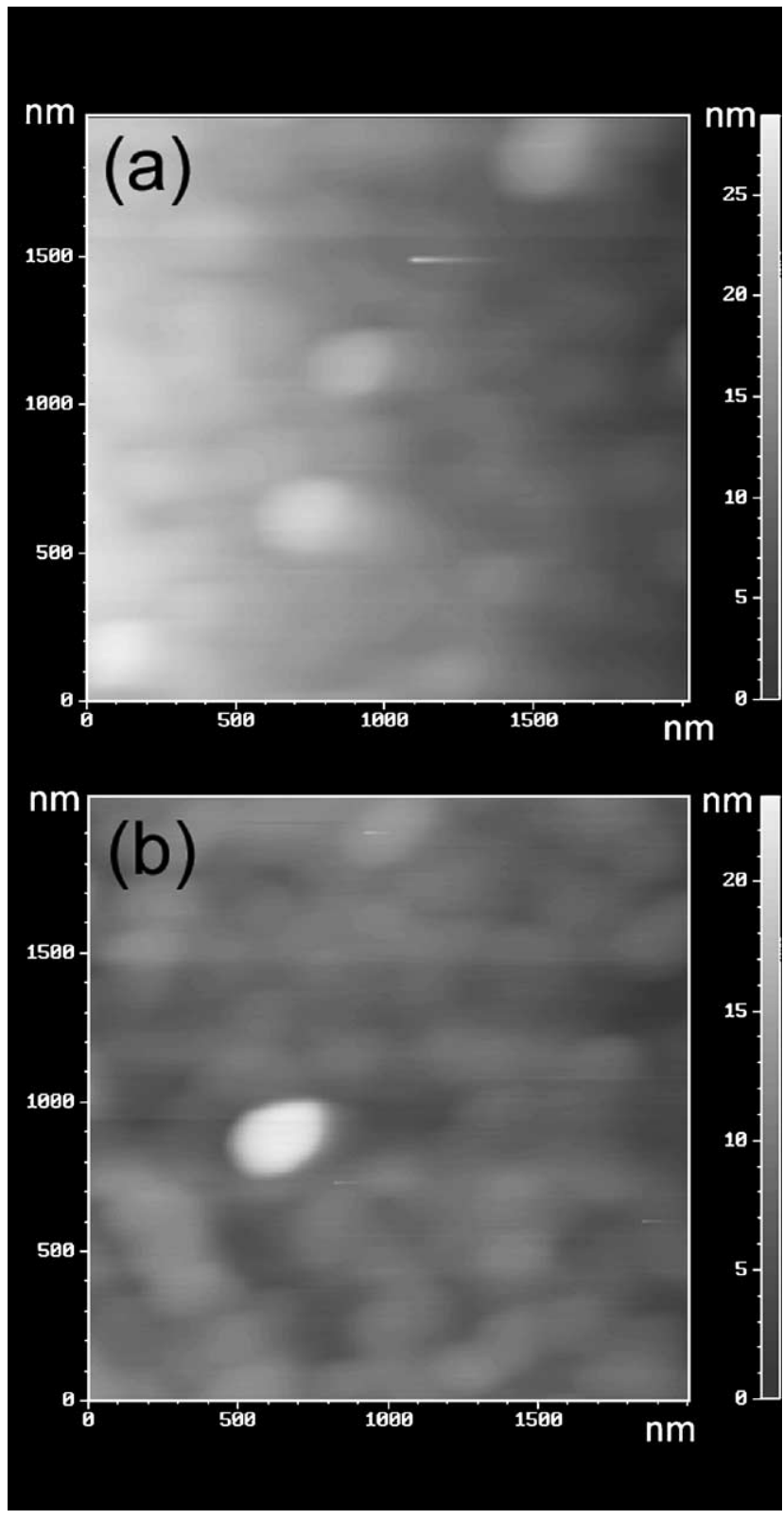

Fig. 6. The AFM topographies of the pristine PMMA substrate (a) and the surface after LAH reduction (b).

rim for extruded PMMA while a flat rim is observed for cast PMMA. The height of the bulge for extruded PMMA is about $10 \mu \mathrm{m}$. Although the bump allows successful sealing of the channels [4], the channel cross section may be distorted because of the refill during bonding. This raises the risk of blocking the fluid flow when a complex pattern is fabricated on the substrate. We therefore decided to use cast PMMA for further experiments.

The rough surface after laser machining has been reported recently [4]. Similar results have been observed in our study. However, the smoothness is greatly enhanced when the laser-machined surface is subjected to thermal annealing. The roughness of pristine cast PMMA is $0.89 \pm 0.10 \mathrm{~nm}$
(RMS) determined by AFM (Fig. 6(a)) whereas the roughness for the initially machined surface is about $5-10 \mu \mathrm{m}$ estimated from the SEM image (Fig. 5(a)). The roughness greatly decreases from several microns to $2.04 \pm 0.61 \mathrm{~nm}$ (RMS) after thermal annealing (Fig. 5(b)). The smoothness is comparable to that of pristine substrate. The roughness of the pristine PMMA sheet shows a slight increase to $1.52 \pm 0.63 \mathrm{~nm}$ (RMS) after LAH treatment (Fig. 6(b)). The increase may results from the attack by LAH or the solvent, ether, used in the reaction. The minute increase suggests that the substrate swelling does not occur. In addition, the roughening effect does not induce visual degradation and the substrate is transparent since the roughness is negligibly small compared to the wavelength of the visible light. The smooth surface thus obtained can minimize light scattering and hence enhance the signal to noise ratio when optical detection is to be used on the chip. The smooth surface also reduces the surface adsorption that can cause contamination and band broadening in separation reaction, e.g. capillary electrophoresis.

\subsection{Universal modification of PMMA surface}

The surface chemistry modification of PMMA has been sporadically reported previously $[6,16]$. The method developed in our group does not require extensive solvent drying procedures. Straightforward chemical reduction of the ester group on the PMMA surface by LAH produces hydroxyl groups $(-\mathrm{OH})$. The functional group is associated to widely used silanization chemistry. Hundreds of organosilanes bearing various organic functional groups are commercially available. Several physical and spectroscopic methods have been utilized to confirm the successful surface reduction.

As a preliminary test for the surface modification, the contact angle shows dramatic change from hydrophobic to hydrophilic surface when pristine PMMA is treated with LAH. Further examination is done by ATR-FTIR and XPS.

The infrared spectra of the PMMA are acquired by ATR-FTIR (Fig. 6) to infer the molecular structure on the surface. For characteristic peaks below $2000 \mathrm{~cm}^{-1}$, our spectra show very similar results to those reported in the literature [17]. The stretching vibrations of $\mathrm{C}=\mathrm{O}$ and $\mathrm{C}-\mathrm{O}-\mathrm{C}$ show similar frequency and relative intensity. However, a prominent broad peak around $3450 \mathrm{~cm}^{-1}$ characteristic of -OH stretching is observed for PMMA sheet after LAH reduction $[18,19]$. Since baking is performed before taking the ATR-FTIR spectra, the broad -OH stretching absorption is unlikely to be caused by surface bound water molecules. In addition, the spectrum remains unchanged after 3 months of storage at room temperature. This result suggests that the modification is permanent. It is also observed that the intensity of the $3450 \mathrm{~cm}^{-1}$ band compared to the characteristic carbonyl band around $1730 \mathrm{~cm}^{-1}$ is relatively weak. This does not necessarily indicate the completeness of reduction. The molecules that contribute to the ATR-FTIR spectra 


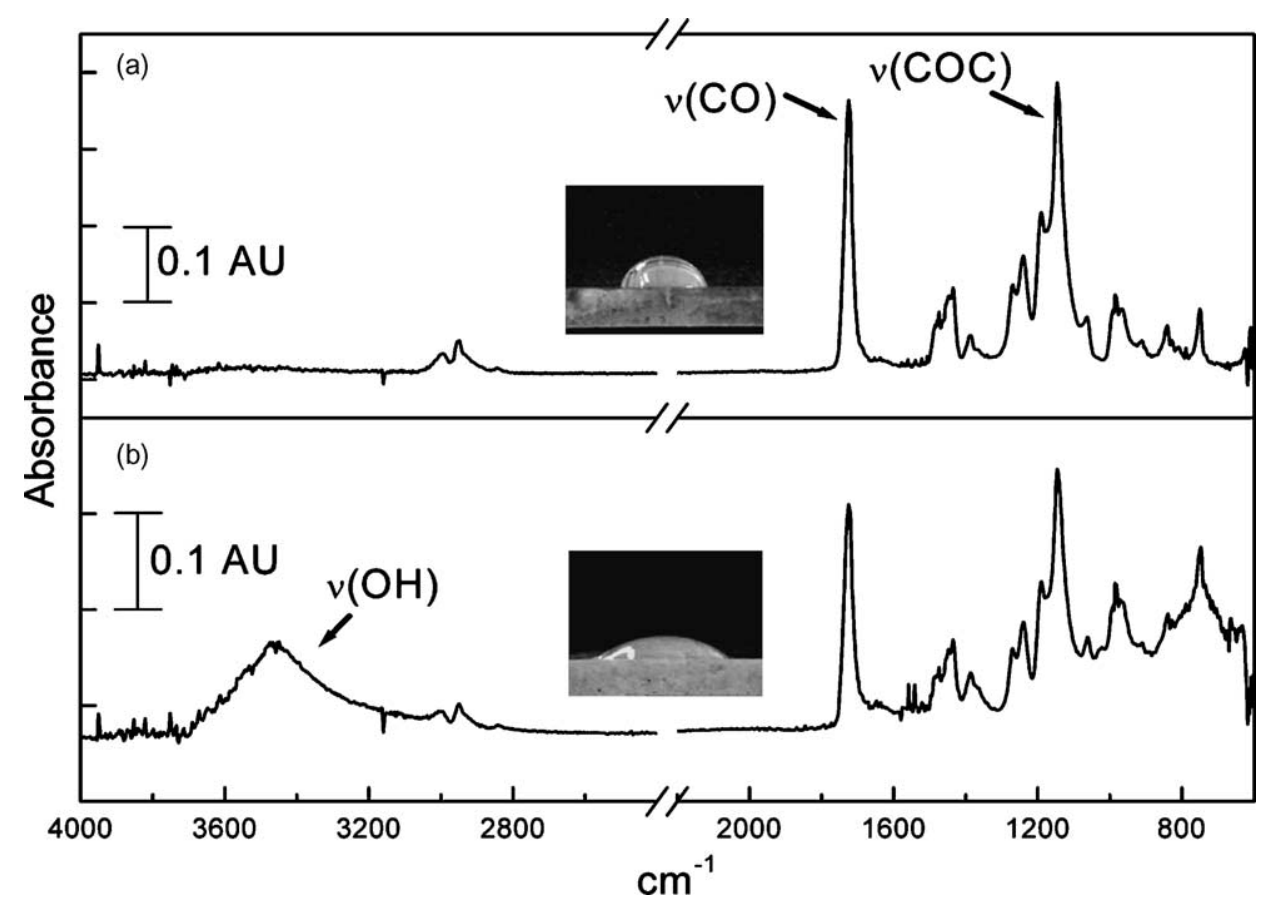

Fig. 7. ATR-FTIR spectra of the pristine (a) and the LAH treated (b) PMMA surface. The insets show the corresponding pictures from the measurement of contact angle.

may have a depth of several microns while the reduction occurs only for surface ester groups. Therefore, the extent of reduction cannot be determined by ATR-FTIR spectra alone. However, it can be estimated that in the single layer of surface molecule the extent of modification should be larger than that revealed in the relative peak intensity of the ATR-FTIR spectra.

It is important to have homogeneous modification on the PMMA surface for practical applications. For a preliminary examination, we have measured contact angles on several spots of the LAH-reduced PMMA. Similar hydrophilic results have been obtained (data not shown).

For further introduction of various organic functional groups, several organosilanes are applied to LAH-reduced PMMA surface. Whether the organosilane is incorporated onto the PMMA surface is examined by XPS. In Fig. 8, characteristic energy corresponding to surface element is indicated. As shown in Fig. 8(a), no heteroatoms except oxygen present on PMMA surface when pristine PMMA is reacted with (3-aminopropyl)trimethoxysilane. The absence of heteroatoms in Fig. 8(a) indicates that no physical adsorption of the organosilane occurs on pristine PMMA surface. Characteristic elements, F, N, and S, showing the successful incorporation of organosilane is clearly observed in Fig. 8(b)-(d) when the LAH treated PMMA is reacted with $1 \mathrm{H}, 1 \mathrm{H}, 2 \mathrm{H}, 2 \mathrm{H}$-perfluorodecyltriethoxysilane, (3-aminopropyl)trimethoxysilane, and 3-mercaptotrimethoxysilane, respectively. These results indicate that the incorporation of the organosilane on the PMMA surface only occurs after LAH reduction. The very weak Si peaks in Fig. 8(b) is due to the fact that the relative atom number of $\mathrm{F}$ to $\mathrm{Si}$ is 17:1 for the perfluorodecyltriethoxysilane. Furthermore, F has higher absorption factor than the Si does and hence much higher intensity is observed. Since the surface roughness of the PMMA only increases by $0.6 \mathrm{~nm}$ when treated with LAH (Fig. 6), it is unlikely that the silane incorporation is due to physical adsorption. We conclude that the incorporation results from the covalent bonding.

The Fig. 8(c) is taken inside a trench. The result shows no apparent difference to the XPS spectra taken on a flat surface. In addition, the immobilized silane is stable in the thermal condition used in bonding procedure since the elevated temperature $\left(120^{\circ} \mathrm{C}\right)$ used in glass silanization does not affect the stability of the silane [20]. Therefore, we conclude that the surface modification should persist inside sealed PMMA channels.

The organosilane modification largely expands the application of the PMMA as a biochip substrate. The perfluoroalkylation modification can be used for surface passivation of PMMA to prevent adsorption. The aminoalkylation and mercaptoalkylation have been used for biomolecule immobilization [20-23]. In addition to demonstrating the capability of the diverse chemical modification, the successful incorporation of the organosilane also suggests the presence of $-\mathrm{OH}$ groups on the PMMA surface after the LAH reduction.

Organosilanes have been widely used for glass surface modification. In addition to those used in this work, organosilanes bearing various functional groups such as, alkene, glycidyloxy, isocyanto, haloalkane, etc. are commercially available. The silane undergoes condensation reaction with silanol group $(-\mathrm{Si}-\mathrm{OH})$ on the glass surface $[24,25]$ and becomes immobilized on the substrate surface. 


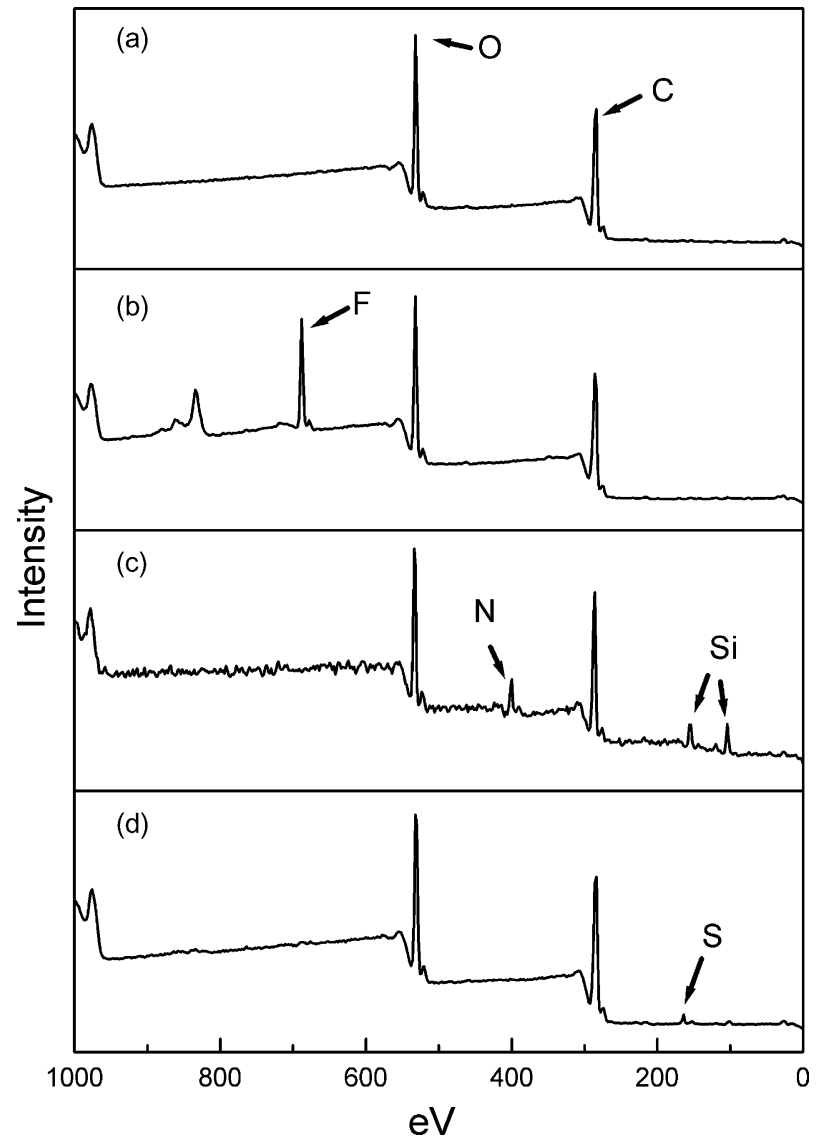

Fig. 8. XPS spectra of various organosilane modified surfaces. Part (a) shows the spectrum of the pristine PMMA after treatment with (3-aminopropyl)trimethoxysilane. Parts (b), (c), and (d) show the spectra of the LAH treated PMMA substrates reacted with $1 \mathrm{H}, 1 \mathrm{H}, 2 \mathrm{H}, 2 \mathrm{H}$-perfluorodecyltriethoxysilane, (3-aminopropyl)trimethoxysilane, and 3-mercaptotrimethoxysilane, respectively.

Organosilane is widely used in the application of DNA microarray and protein microarray for DNA/protein immobilization or for surface passivation to prevent non-specific binding. Although most of the existing examples describe silanization on the glass substrate, other substrates such as plastic are also applicable as long as hydroxyl groups are present on the surface [26]. The silane condensation reaction is usually performed by incubation at room temperature followed by curing in vacuum at slightly elevated temperature. The process provides an efficient method for plastic surface modification. When combined with the organosilane condensation reaction, the LAH reduction of PMMA has produced a surface suitable for further introduction of numerous functional groups, which in turn can be used in biological applications. The modification has thus provided a universal base for diverse applications.

\section{Conclusions}

In this work, we have demonstrated an economic and flexible PMMA micromachining pipeline suitable for swift prototyping and production. We have overcome the general problem of rough surface after laser machining. A simple thermal annealing process has been developed to enhance the smoothness of the initially rough machined surface to be nearly comparable to that of a pristine PMMA sheet. The quality of the surface has been examined by SEM and AFM. A universal surface modification method allowing a subsequent silanization reaction has been described and confirmed by ATR-FTIR. The incorporation of several organic functional groups has been demonstrated by applying several organosilanes to the modified PMMA. The successful organosilane incorporation has been verified by XPS. The overall procedures include not only the microstructure machining but also the post-processing basis for biochemical and biological applications.

\section{Acknowledgements}

We would like to thank National Science Council (NSC 91-2113-M-001-051) and Academia Sinica, Taiwan for their financial support. We also thank Dr. Peilin Chen, Dr. Pei Kuen Wei, Mr. Shie Li Chou and Miss Chon Wen Kuo for the assistance in AFM measurement. We also thank Miss Hsiang-Ping Wen for the assistance in XPS measurement.

\section{References}

[1] H. Becker, U. Heim, Hot embossing as a method for the fabrication of polymer high aspect ratio structures, Sens. Actuators A: Phys. 83 (2000) 130-135.

[2] L. Martynova, L.E. Locascio, M. Gaitan, G.W. Kramer, R.G. Christensen, W.A. MacCrehan, Fabrication of plastic microfluid channels by imprinting methods, Anal. Chem. 69 (1997) 4783-4789.

[3] M.A. Roberts, J.S. Rossier, P. Bercier, H. Girault, UV laser machined polymer substrates for the development of microdiagnostic systems, Anal. Chem. 69 (1997) 2035-2042.

[4] H. Klank, J.P. Kutter, O. Geschke, $\mathrm{CO}_{2}$-laser micromachining and back-end processing for rapid production of PMMA-based microfluidic systems, Lab on a Chip 2 (2002) 242-246.

[5] R.T. Morrison, R.N. Boyd, Organic Chemistry, 5th ed., Allyn and Bacon, Boston, 1987, Chapter 24, pp. 875-877.

[6] A.C. Henry, T.J. Tutt, M. Galloway, Y.Y. Davidson, C.S. McWhorter, S.A. Soper, R.L. McCarley, Surface modification of poly(methyl methacrylate) used in the fabrication of microanalytical devices, Anal. Chem. 72 (2000) 5331-5337.

[7] P.O. Brown, D. Botstein, Exploring the new world of the genome with DNA microarrays, Nat. Genet. Suppl. 21 (1999) 33-37.

[8] M.L. Bulyk, E. Gentalen, D.J. Lockhart, G.M. Church, Quantifying DNA-protein interactions by double-stranded DNA arrays, Nat. Biotechnol. 17 (1999) 573-577.

[9] A.Q. Emili, G. Cagney, Large-scale functional analysis using peptide or protein arrays, Nat. Biotechnol. 18 (2000) 393-397.

[10] H. Ge, UPA, a universal protein array system for quantitative detection of protein-protein, protein-DNA, protein-RNA and protein-ligand interactions, Nucl. Acids Res. 28 (2000) e3.

[11] M.U. Kopp, A.J. de Mello, A. Manz, Chemical amplification: continuous-flow PCR on a chip, Science 280 (1998) 1046-1048.

[12] E.A. Schilling, A.E. Kamholz, P. Yager, Cell lysis and protein extraction in a microfluidic device with detection by a fluorogenic enzyme assay, Anal. Chem. 74 (2002) 1798-1804. 
[13] C.T. Culbertson, S.C. Jacobson, J.M. Ramsey, Microchip devices for high-efficiency separations, Anal. Chem. 72 (2000) 5814-5819.

[14] R.D. Oleschuk, L.L. Shultz-Lockyear, Y. Ning, D.J. Harrison, Trapping of bead-based reagents within microfluidic systems: on-chip solid-phase extraction and electrochromatography, Anal. Chem. 72 (2000) 585-590.

[15] C. Yu, M.H. Davey, F. Svec, J.M.J. Fre'chet, Monolithic porous polymer for on-chip solid-phase extraction and preconcentration prepared by photoinitiated in situ polymerization within a microfluidic device, Anal. Chem. 73 (2001) 5088-5096.

[16] B. Karandikar, J. Puschett, K. Matyjaszewski, Homogeneous and heterogeneous modification of poly(methyl methacrylate) with ethylene diamine, Polym. Prep. 30 (1989) 250-251.

[17] R.M. Silverstein, G.C. Bassler, T.C. Morrill, Spectrometric identification of Organic Compounds, 4th ed., Wiley, New York, NY, 1981, p. 121.

[18] R.M. Silverstein, G.C. Bassler, T.C. Morrill, Spectrometric identification of Organic Compounds, 4th ed., Wiley, New York, NY, 1981, p. 112.

[19] C. Wochnowski, S. Metev, G. Sepold, UV-laser-assisted modification of the optical properties of polymethylmethacrylate, Appl. Surf. Sci. 154-155 (2000) 706-711.

[20] N. Zammatteo, L. Jeanmart, S. Hamels, S. Courtois, P. Louette, L. Hevesi, J. Remacle, Comparison between different strategies of covalent attachment of DNA to glass surfaces to build DNA microarrays, Anal. Biochem. 280 (2000) 143-150.

[21] Y.H. Rogers, P. Jiang-Baucom, Z.J. Huang, V. Bogdanov, S. Anderson, M.T. Boyce-Jacino, Immobilization of oligonucleotides onto a glass support via disulfide bonds: a method for preparation of DNA microarrays, Anal. Biochem. 266 (1999) 23-30.

[22] L.A. Chrisey, G.U. Lee, C.E. O'Ferrall, Covalent attachment of synthetic DNA to self-assembled monolayer films, Nucl. Acids Res. 24 (1996) 3031-3039.

[23] A. Kumar, O. Larsson, D. Parodi, Z. Liang, Silanized nucleic acids: a general platform for DNA immobilization, Nucl. Acids Res. 28 (2000) e71.

[24] I. Shimizu, A. Yoshino, H. Okabayashi, E. Nishio, C.J. O'Connor, Kinetics of interaction of 3-aminopropyltriethoxysilane on a silica gel surface using elemental analysis and diffuse reflectance infrared Fourier transform spectra, J. Chem. Soc., Faraday Trans. 93 (1997) 1971-1979.
[25] C.M. Halliwell, A.E.G. Cass, A factorial analysis of silanization conditions for the immobilization of oligonucleotides on glass surfaces, Anal. Chem. 73 (2001) 2476-2483.

[26] J.C. Lin, C.H. Wu, Surface characterization and platelet adhesion studies on polyurethane surface immobilized with $\mathrm{C}_{60}$, Biomaterials 20 (1999) 1613-1620.

\section{Biographies}

Dr. Ji-Yen Cheng received his bachelor's degree in 1990 from National Taiwan University, Taiwan. He later received his master, and $\mathrm{PhD}$ degree in chemistry in 1992 and 1998 from the same university. After graduation, he entered Institute of Biomedical Sciences, Academia Sinica, Taiwan for post-doctoral training in DNA microarray under the mentor of Dr. Konan Peck. Dr. Cheng's expertise includes high-throughput DNA synthesis, oligonucleotide microarray and microfabrication. He is now an assistant research fellow in the Institute for Applied Science and Engineering Research, Academia Sinica, Taiwan. His current research interests focus on the fabrication and biomedical applications of microfluidic chip.

Mr. Cheng-Wey Wei graduated from National Chung Hsing University, Taiwan and received bachelor degree in chemical engineering (1998). He then entered National Taiwan University (NTU) where he obtained master's degree in chemical engineering (2000). He is now a PhD student in the Institute of Biomedical Engineering, NTU. His research interest is in plastic microfluidic devices and related biomedical applications.

Mr. Kai-Hsiung Hsu graduated from the Department of Physics, National Sun-Yat-Sen University, Taiwan in 1996 and received master's degree from the Institute of Optical Electronics, National Chiao Tung University, Taiwan. His research interest is in microfabrication and microelectromechanical system (MEMS).

Prof. Tai-Horng Young is a professor in the Institute of Biomedical Engineering, National Taiwan University, Taiwan. He graduated from the Department of Chemical Engineering, National Taiwan University and received his bachelor degree, master degree and $\mathrm{PhD}$ degree in 1984, 1986 and 1991, respectively. His research interests include polymeric biomaterials and tissue engineering. 